\title{
Effects of Varying Gravity Levels on fNIRS Headgear Performance and Signal Recovery
}

\author{
Jeffrey R. Mackey ${ }^{1}$ \\ Vantage Partners, LLC, Brook Park, Ohio, 44142 \\ Angela R. Harrivel ${ }^{2}$, Grigory Adamovsky ${ }^{3}$, Beth E. Lewandowski ${ }^{4}$ \\ NASA Glenn Research Center, Cleveland, Ohio, 44135 \\ Daniel J. Gotti ${ }^{5}$, Padetha Tin ${ }^{6}$ \\ National Center for Space Exploration Research, Cleveland, Ohio, 44135 \\ and \\ Bertram M. Floyd ${ }^{7}$ \\ Sierra Lobo, Cleveland, Ohio, 44135
}

\begin{abstract}
This paper reviews the effects of varying gravitational levels on functional Near-Infrared Spectroscopy (fNIRS) headgear. fNIRS systems quantify neural activations in the cortex by measuring hemoglobin concentration changes via optical intensity. Such activation measurement allows for the detection of cognitive state, which can be important for emotional stability, human performance and vigilance optimization, and the detection of hazardous operator state. The technique depends on coupling between the fNIRS probe and user's skin. Such coupling may be highly susceptible to motion if probe-containing headgear designs are not adequately tested. The lack of reliable and self-applicable headgear robust to the influence of motion artifact currently inhibits its operational use in aerospace environments. Both NASA's Aviation Safety and Human Research Programs are interested in this technology as a method of monitoring cognitive state of pilots and crew.
\end{abstract}

\section{Nomenclature}

$\begin{array}{ll}a c & =\text { alternating current } \\ a x & =\text { acceleration level in the } x \text { direction } \\ a y & =\text { acceleration level in the } y \text { direction } \\ a z & =\text { acceleration level in the } z \text { direction } \\ d c & =\text { direct current } \\ \text { fNIRS } & =\text { functional near infrared spectroscopy } \\ G R I N & =\text { graded index } \\ g x & =\text { gravitational force in the } x \text { direction } \\ g y & =\text { gravitational force in the } y \text { direction } \\ g z & =\text { gravitational force in the } z \text { direction }\end{array}$

\footnotetext{
${ }^{1}$ Research Engineer, 3000 Aerospace Parkway, Brook Park, OH 44142/MS GESS-3.

${ }^{2}$ Research Engineer, 21000 Brookpark Rd, Cleveland, OH 44135/MS 110-2, AIAA Senior Member.

${ }^{3}$ Sr. Research Engineer, 21000 Brookpark Rd, Cleveland, OH 44135/MS 77-1, AIAA Associate Follow.

${ }^{4}$ Biomedical Research Engineer, 21000 Brookpark Rd, Cleveland, OH 44135/MS 110-3.

${ }^{5}$ Mechanical Engineer, 21000 Brookpark Rd, Cleveland, OH 44135, MS 110-3.

${ }^{6}$ Staff Scientist, 21000 Brookpark Rd, Cleveland, OH 44135, MS 110-3.

${ }^{7}$ Engineering Lead, 21000 Brookpark Rd, Cleveland, OH 44135/MS 77-3.
} 


\section{Introduction}

$\mathrm{A}$ vation Safety research is being conducted by the NASA Glenn Research Center's Aeronautics Directorate regarding the use of functional near-infrared spectroscopy (fNIRS) technology to detect cognitive state as part of an overall Vehicle Systems Safety Technologies (VSST) Project goal to provide a 75\% improvement over current methods in crew decision-making during off-nominal conditions. This paper examines the development of novel headgear and its characterization under variable gravity levels. In particular, the optical transmitter/receiver assemblies that can be incorporated into an fNIRS system for use in real-time cognitive state detection in the cockpit were characterized during several parabolic aircraft flights. The goal of our experiment was to characterize and evaluate the newly developed fNIRS instrumentation under such rapidly changing gravitational levels in an actual flight environment.

Custom optical probes were fabricated at Glenn Research Center and tested in various configurations, using both silica and plastic optical fiber for the amplitude-modulated laser light delivery and collection at 690 and 830 nanometer wavelengths. The hardware used in the fNIRS variable gravity (zero to 1.8 times Earth gravity) experiment consisted of custom-designed optical instrumentation, probe-holding headgear with tension adjustment, and a light scattering test phantom simulating gray matter found in the human brain. The modulation reference and raw scattered signals were received by photomultiplier tubes and the raw signals were digitized and recorded on a digital oscilloscope. These signals were simultaneously passed through electronic lowpass filters and lock-in amplifiers to record their $d c$ - and $a c$-components using a data acquisition system for post-flight data analysis. Headgear strap tension levels were varied, and the optical signal intensity as a function of both the tension level and gravitational level was analyzed to determine appropriate forces that would be imposed on the scalp for the reduction of artifact without inducing appreciable pain. Such data is vital to decouple the mechanical effects induced by varying gravitational levels and similarly-induced psychological and physiological artifacts from the cognitive response to the functional task of interest. Therefore, the characterization of the instrumentation with respect to gravity level obtained in this study allows for design ameliorations suitable for implementation using human participants in the near future.

\section{Background}

Despite the continuing improvement of various research laboratory-based and commercial instrumentation, hardware currently used to attach optical fibers to and couple optical signals to the scalp, fNIRS behind the hairline ${ }^{1}$ is bulky, uncomfortable to painful, susceptible to motion artifact and interference from the hair, not expeditiously self-applicable and not readily integrated with existing environments ${ }^{2,3}$ (for example, cockpits and pilot headsets). Reducing such difficulties consistently encountered by those in both industry and academia who uses fNIRS is a design-driven aim. The success of these efforts to produce "next generation" fNIRS headgear would bring the benefits of the fNIRS technique out of the controlled laboratory and into clinical and operational environments. The full-up instrumentation that was tested and reported upon in this paper includes optical, electronic and medical grade components. This full system is required to support the development of such headgear.

In the NASA Aviation Safety Program, the fNIRS technology is being developed as part of an overall Vehicle Systems Safety Technologies (VSST) Project goal to provide a 75\% improvement in crew decision-making under off-nominal conditions. The fNIRS project directly addresses the NASA VSST goal because the ultimate goal of the fNIRS project is to determine the cognitive state of the pilot ${ }^{4}$.

For long duration space missions, prolonged physiological and psychological effects on crew members are major safety and health concerns ${ }^{5}$. The need for the evaluation of such effects on crew personnel during space flight are outlined in the NASA OCT Roadmap Technology Area 06, Human Health, Life Support and Habitation Systems (HLHS), Subtopic Human Health performance. Furthermore, exposure to gravitational changes through planetary orbital maneuvering may also affect astronaut and pilot performance. fNIRS provides a means to evaluate such effects.

In military activities, there is a need to collect physiologic data associated with mild traumatic brain injury and post-traumatic stress disorder near the forward edge of the battle area. Of the potential methods, only the electroencephalogram (EEG) and functional Near Infrared Spectroscopy (fNIRS) have the potential to be made portable and inexpensive ${ }^{2}$. The DOD has recently sponsored a SBIR Phase I award (Award ID 97706 granted in 2010) to integrate elements of a commercial fNIRS sensor into the body of the most advanced EEG sensor currently available.

fNIRS has been performed on past reduced gravity flights, but not with our focus on the headgear coupling efficiency. To date, reduced gravity fNIRS has been done on the forehead only ${ }^{6,7}$. Also, our short source-detector 
separation sensors allow for the measurement of systemic blood volume changes, which is a significant physiological confound ${ }^{7,8,9}$ during gravity level changes.

\section{Experiment Description}

The experimental fNIRS configuration consisted of a fiber-optic light delivery system and a fiber-optic light receiver system as illustrated in Fig. 1. For testing and instrument characterization purposes, the light delivery system was streamlined to combine light from two different wavelength laser systems and power two different optical transmission systems delivering $830 \mathrm{~nm}$ and $690 \mathrm{~nm}$ light to the skin. These transmission systems are sometimes referred to as "optodes".

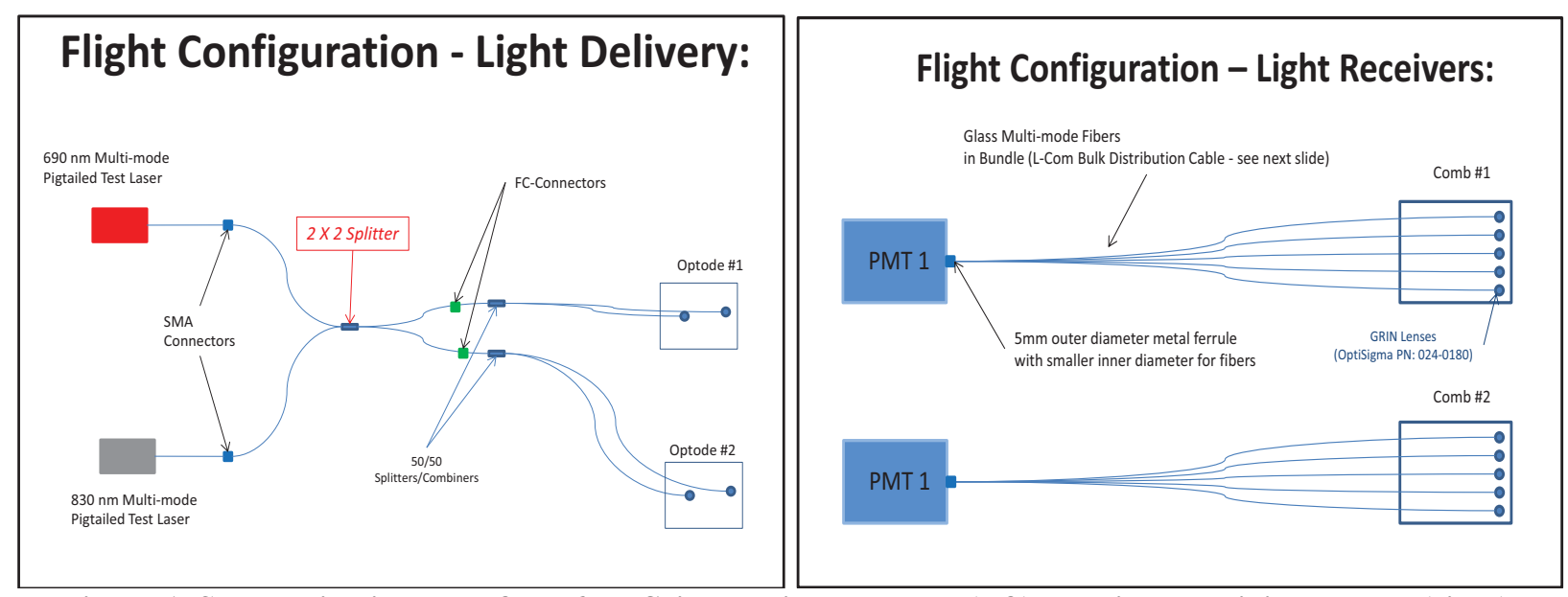

Figure 1. Schematic diagram of the fNIRS light delivery system (left) and light receiving system (right).

As illustrated in Fig. 2, the transmitters and receivers were custom fabricated using a GRIN lens coupled to an optical fiber'. The initial designs used silica fiber having a 125 micrometer total diameter (core plus cladding). Such a configuration is very flexible and allows for a small bend radius in the optical fiber. Other designs were similar, but used a larger diameter plastic optical fiber (POF) manufactured by Chromis Fiberoptics, Inc., Warren, NJ. The Chromis POF adequately transmitted both the $690 \mathrm{~nm}$ and the $830 \mathrm{~nm}$ laser wavelengths while other POF types provided too much attenuation of the $830 \mathrm{~nm}$ light over the 10 meter fiber length desired for the fNIRS system.

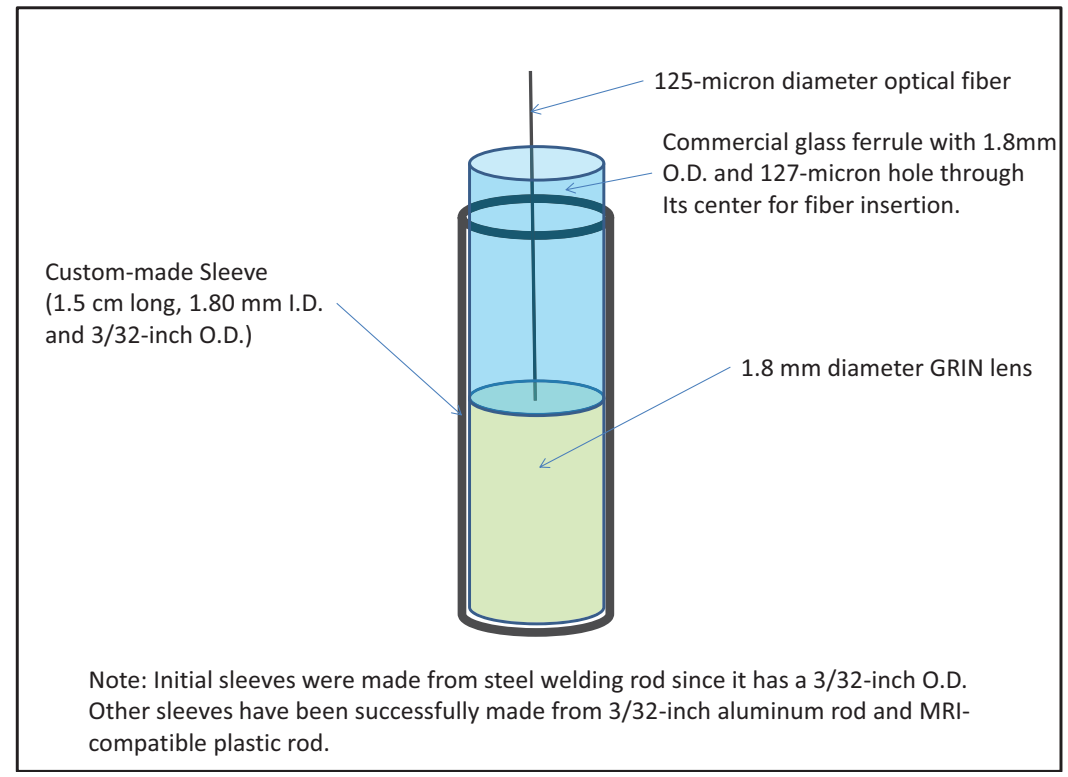

Figure 2. fNIRS GRIN lens to fiber coupling concept.

American Institute of Aeronautics and Astronautics 
Different GRIN lenses were used in the transmitters than in the receivers. The transmitter lenses were chosen to optimize focusing of the $690 \mathrm{~nm}$ laser wavelength to approximately $12 \mathrm{~cm}$ from the exit aperture of the lens in order to better penetrate the low-scattering cerebral-spinal fluid barrier. The transmitter lens approximately collimates the $830 \mathrm{~nm}$ laser light. The receiver lenses were chosen to maximize the light collection angle for the $690 \mathrm{~nm}$ light scattered through the gray matter or in our case, gray matter phantom material.

\section{A. Flight Configuration}

The signal generation for the fNIRS research flight system was accomplished using mostly commercial off the shelf electronics. Two laser diodes having wavelengths of $690 \mathrm{~nm}$ and $830 \mathrm{~nm}$ respectively were permanently pigtailed to multi-mode 62.5 micrometer core silica fibers having a NA of 0.36 . The coupling efficiency was approximately $40 \%$ for each of the laser systems. The lasers were powered by an OEM laser driver capable of operating and amplitude modulating both lasers at various frequencies between $1 \mathrm{~Hz}$ and $500 \mathrm{kHz}$. A baseline frequency of $5 \mathrm{kHz}$ was chosen because this frequency is within the range of the bench top lock-in amplifiers used to recover the ac signal component. Furthermore, the $5 \mathrm{kHz}$ signal is easily filtered by the lowpass filter set to a 300 $\mathrm{Hz}$ cutoff to recover the dc signal component. The lock-in amplifiers provided a crystal oscillator output, which was used to drive the laser diode driver modulation circuit. The laser fibers were connected to a 2 X 2 multimode fiberoptic splitter so that both the $690 \mathrm{~nm}$ and the $830 \mathrm{~nm}$ wavelength was output on both output legs, providing amplitude-modulated signals to two test articles simultaneously. This configuration was used during the research flights to provide the modulated illumination signals to the headgear as well as to the test phantom as illustrated in Fig. 3.

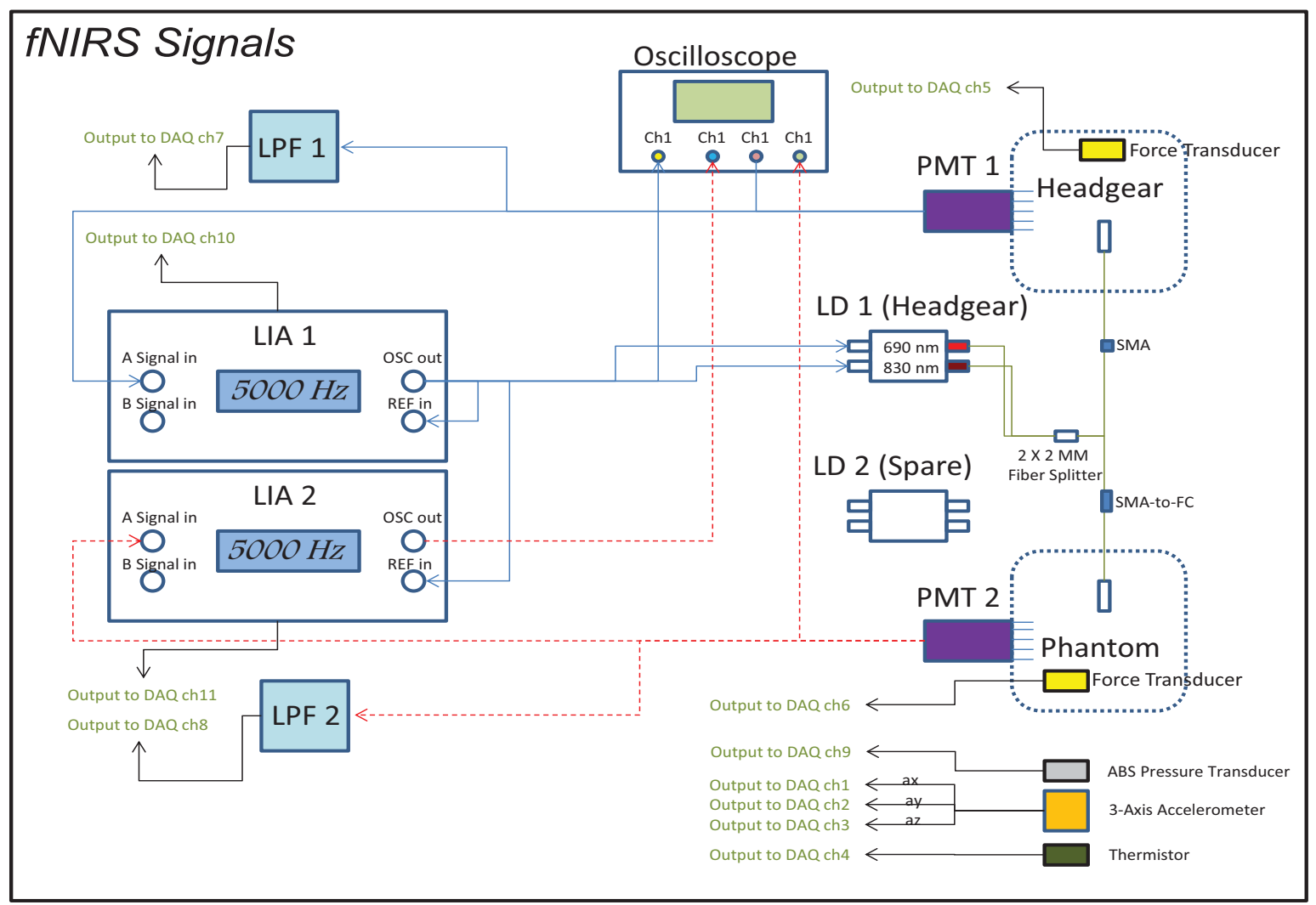

Figure 3. Schematic diagram of the flight experiment fNIRS signal generation and recovery.

The weak scattered optical signals were converted to electronic signals by photomultiplier tubes after entering the pick-up fibers, which transmitted the light through the tube aperture. The electronic signals (voltage) coming from the photomultiplier tubes was split using a coaxial cable splitter and the split signal from each photomultiplier tube was input simultaneously into a channel of a digital oscilloscope and to the lowpass filter and lock-in amplifier to obtain the $d c$ and $a c$ components simultaneously. The outputs from the lowpass filter ( $d c$ signal component) and 
lock-in amplifier (ac signal component) were input to a data acquisition system, which digitized the signals and recorded them for post processing.

All equipment and instrumentation described herein was configured and assembled into a vertical aluminum rack specially fabricated for the NASA KC-135 parabolic aircraft. Since the bolt patterns are the same in the Zero-G B727 aircraft, this rack was chosen for these experiments. To assure laser safety aboard the aircraft, the upper chamber housing the lasers and test articles was enclosed by opaque panels and a hinged door that was interlocked to turn off all lasers when opened. A photograph of the flight experiment test rig is shown in Fig. 4.

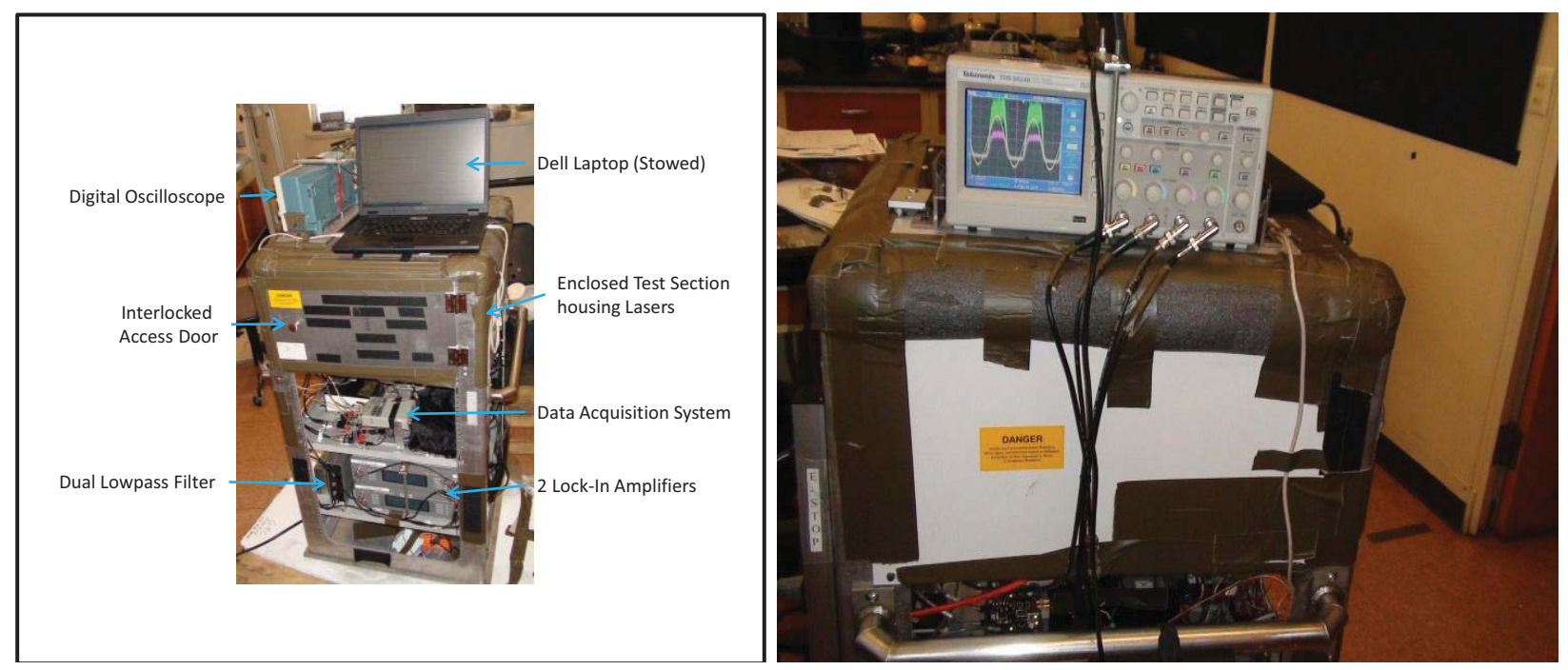

Figure 4. Photographs of fNIRS flight experiment rig configured as flown.

Once all components passed safety inspection, the rig was loaded into the aircraft and flight test were conducted by two fNIRS team members. One researcher operated data acquisition functions using the digital oscilloscope (Fig. 5) while the other researcher operated the headgear and phantom tension mechanisms and monitored the experimental rig instrumentation.

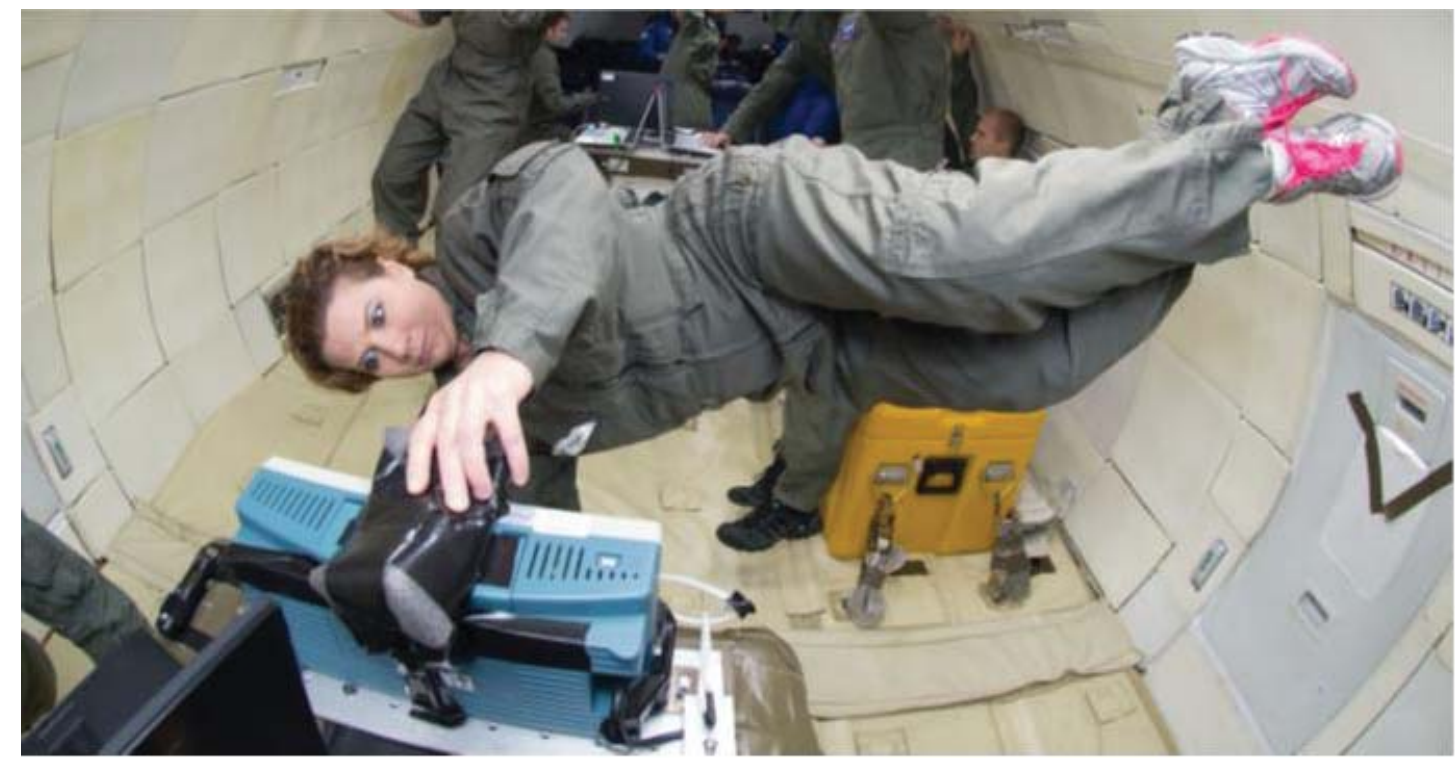

Figure 5. fNIRS Researcher Beth Lewandowski of NASA Glenn Research Center acquiring fNIRS data during microgravity portion of variable gravity research flight.

In all, three different headgear configurations were tested on phantom heads and two different fNIRS optical platform configurations were tested on the gray matter phantom block. The headgear included a semi-rigid parietal 
headgear band, a semi-rigid frontal headgear band and a flexible strap-based headgear that could be used to test either frontal coupling or parietal coupling. The optical platforms tested on the gray matter phantom block included a silica fiber coupled to GRIN lens configuration housed in a custom aluminum fixture and a MRI-compatible POF coupled to GRIN lens configuration housed in a custom semi-rigid rubber fixture. The various test articles are described in the following section.

\section{Experimental Methods}

To generate the scattered light responsible for producing the spectroscopic signal, a tissue phantom having similar absorption and scattering properties to human gray matter was utilized. The aluminum fixture housing the silica fiber optical platform was mounted to the phantom material by a compression mechanism and tension was adjusted via a lead screw (Fig. 6). A calibrated load cell was placed between the lead screw tip and the fNIRS optical platform. During the flight, tension levels were adjusted and measured as a function of time. Synchronous acceleration measurements were also measured as a function of time so that coupling efficiencies could be determined as a function of gravity level and tension.
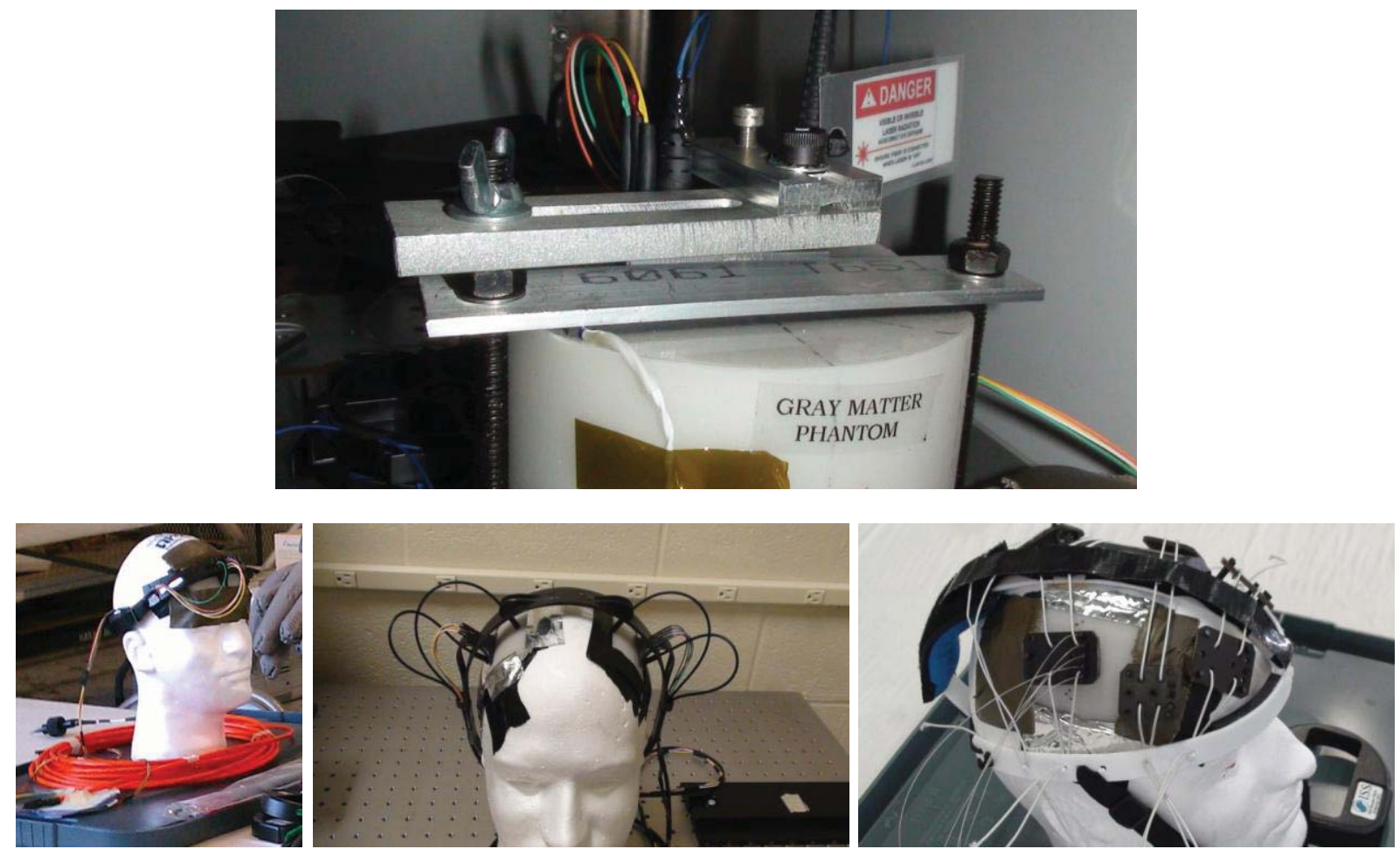

Figure 6. Test article compression mounted to gray matter phantom block. The lead screw was used to adjust coupling force on the optical housing (top). Frontal semi-rigid band headgear (bottom left),

Parietal semi-rigid band headgear (bottom middle) and Parietal rubber holder and elastic strap headgear with POF (bottom right)

For both parietal headgear designs (semi-rigid band and strap), the optical component placements were identical. The mechanical aspects of the head attachment mechanisms were different. One design was shaped like a headband and employed all glass optical fiber. These were designed in-house and 3D printed. They were semi-rigid, and source components were attached with a leaf-spring mechanism. The second design used POF and individual rubber were pieces held on the head with elastic straps. Both designs employed chin straps, and were mounted on a Styrofoam head with embedded gray matter phantom material (Fig. 6). All optical component assembly was accomplished in-house at NASA Glenn Research Center.

The tension adjustment for the band type of headgear was accomplished by simply adjusting the band position. Due to the constraints of the flight experiment enclosure, the semi-rigid headgear strap could not be adjusted in the 
normal fashion by pulling the strap, so strap tension settings were changed using a post and holder configuration shown in Fig. 7.
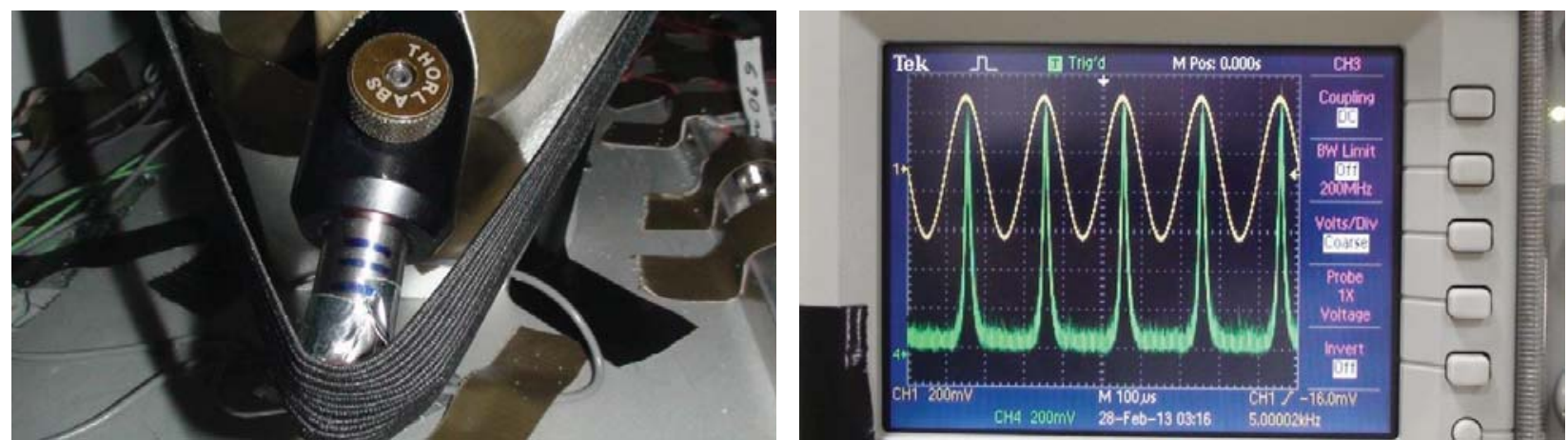

Figure 7. Parietal semi-rigid band headgear (top left) and tension adjustment mechanism with load cell under the strap (top right), band type headgear with POF and rubber optical platform set in parietal configuration (bottom left) and raw signal traces from the modulation driver (yellow trace) and headgear (green trace).

The three headgear designs were tested on different flight days. At least four tension levels between the headgear and the phantom material were tested. The tension was varied during level flight. All tension levels were less than one pound of force on the phantom material, which should be within the comfort range of healthy adults.

Data were recorded at $5.5 \mathrm{~Hz}$ for each tension setting for each design configuration across multiple parabolas. Plastic fiber affords the potential for a less obtrusive, lower profile due to a smaller minimum bend radius, but at the cost of lower intensity throughput. Therefore, intensity signals were normalized to remove differences in absolute magnitude due to plastic and glass optical fiber attenuation.

\section{Results and Discussion}

Results from the five flights are presented and discussed in this section. The first parabolic aircraft flight took place on September 11, 2012. Due to weather conditions, the September 11 flight was the only one conducted during that particular flight week. As a result, the NASA Flight Opportunities Program offered another flight week with parabolic flights occurring on February 26, 27, 28 and March 1, 2013.

\section{DC signal correlation analysis and signal artifact removal correction results:}

The correlations between the force, optical intensity and acceleration (the z-component of the rig acceleration, noted as " $g z$ ") time traces were calculated for varying tension levels for each design (with tension 4 being the highest). These correlations quantify the motion artifacts that occur because as the optical probes move, the detected optical intensity can also change due to detector exposure to low-level ambient light, and to inefficient coupling of light into and out of the phantom material. These inefficiencies are primarily caused by the generation of air gaps between the optical components and the phantom (or skin) surface. These measures and the associated traces are presented for the parietal rubber holder and elastic strap assembly (Fig 8.). The traces were normalized then smoothed over 2 seconds prior to computing the correlation between 1) the detected $d c$ intensity and the rig acceleration, and 2) the detected $d c$ intensity and the force between the rubber holder headgear and the phantom.

The lowest correlation was observed at tension level 3, as can be seen in the upper panel of Fig. 8. This data indicates the least contribution of changes in the mechanical operational environment to the changes in optical intensity. However, motion artifact and oscillations both remain during the low-g portions of the parabolic flight, especially at the transitions in g. Furthermore, the acceleration and force traces were treated as unwanted or nuisance contributions to the intensity signal, and were removed via standard linear regression, shown in the lower panel of Fig. 8. The corrected intensity signal has had varying amounts of the nuisance traces removed by linear subtraction. The amount removed is represented by the Beta multiplier for each of the two traces. The Beta value for the acceleration (" $g z$ ") acts as a metric on the ability of the headgear to reject contributions from the acceleration of the flight rig because its magnitude represents the contribution of that trace to the uncorrected intensity signal. Thus, lower Beta values imply less susceptibility to changes in the mechanical environment. The Beta values were again smallest for the parietal rubber holder headgear, at tension levels 2 and 3 as in the bottom panel of Fig. 8. At local time scales, force and intensity changes were sometimes highly correlated and sometimes not. We recommend 
using acceleration data, and force measurements if possible, as nuisance regressors for intensity signal correction in operational environments.
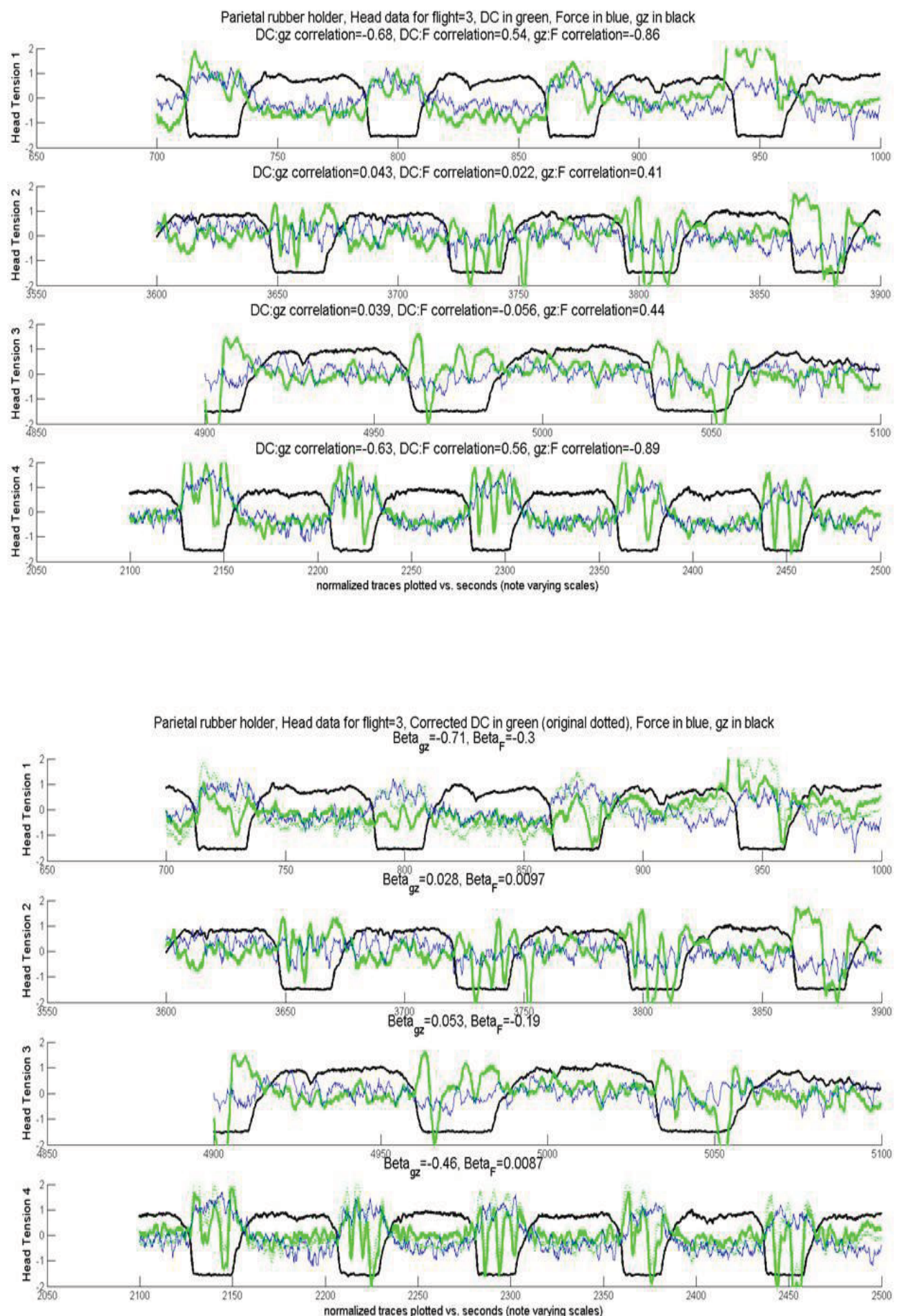

Figure 8. Parietal rubber holder and elastic strap headgear traces: correlations (upper) and corrected traces (lower) 

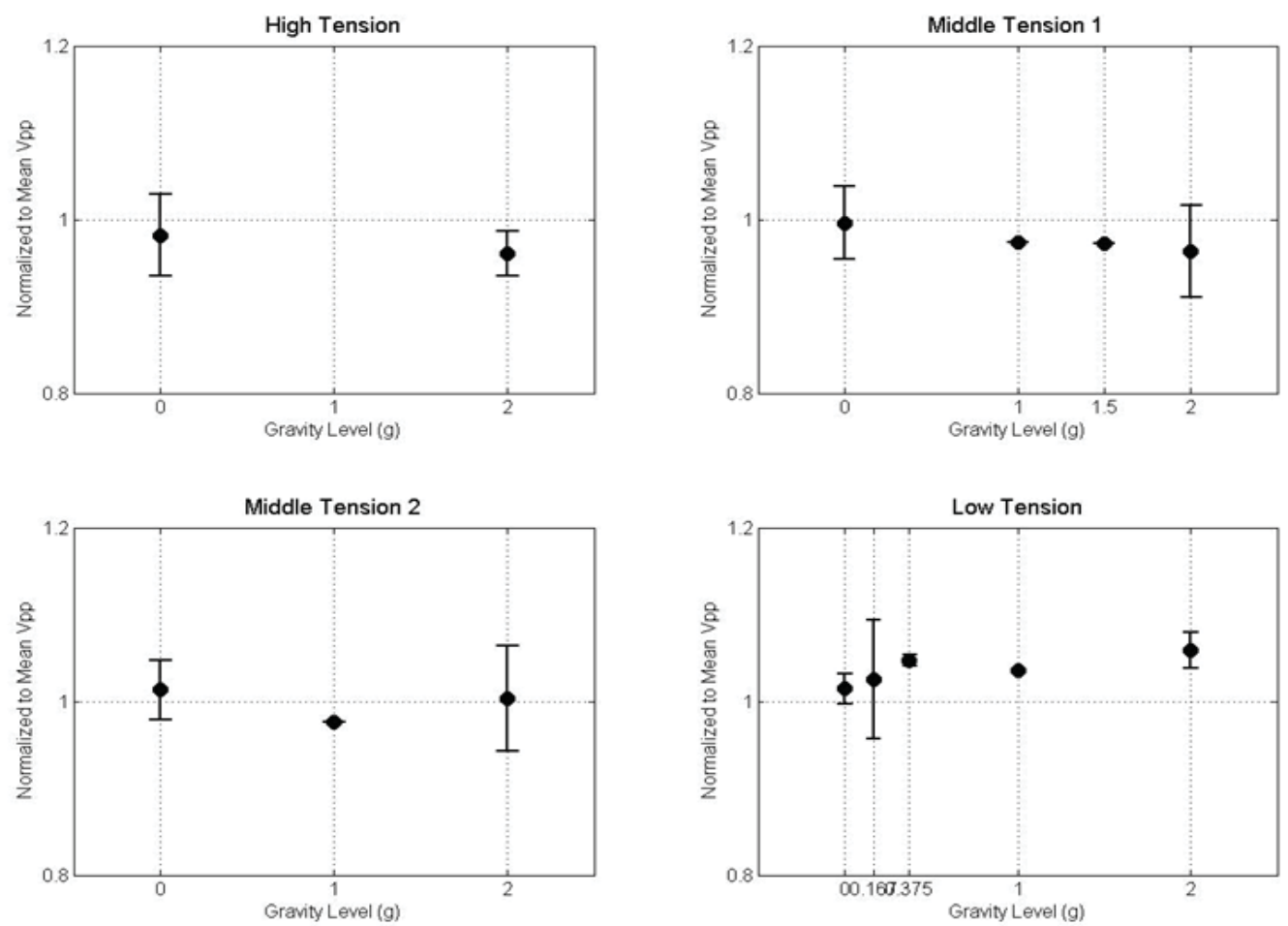

Figure 9. Frontal semi-rigid band headgear (flight day 26, February 2013): Normalized AC signal intensity measured over multiple parabolas plotted vs. gravity level for each tension level.
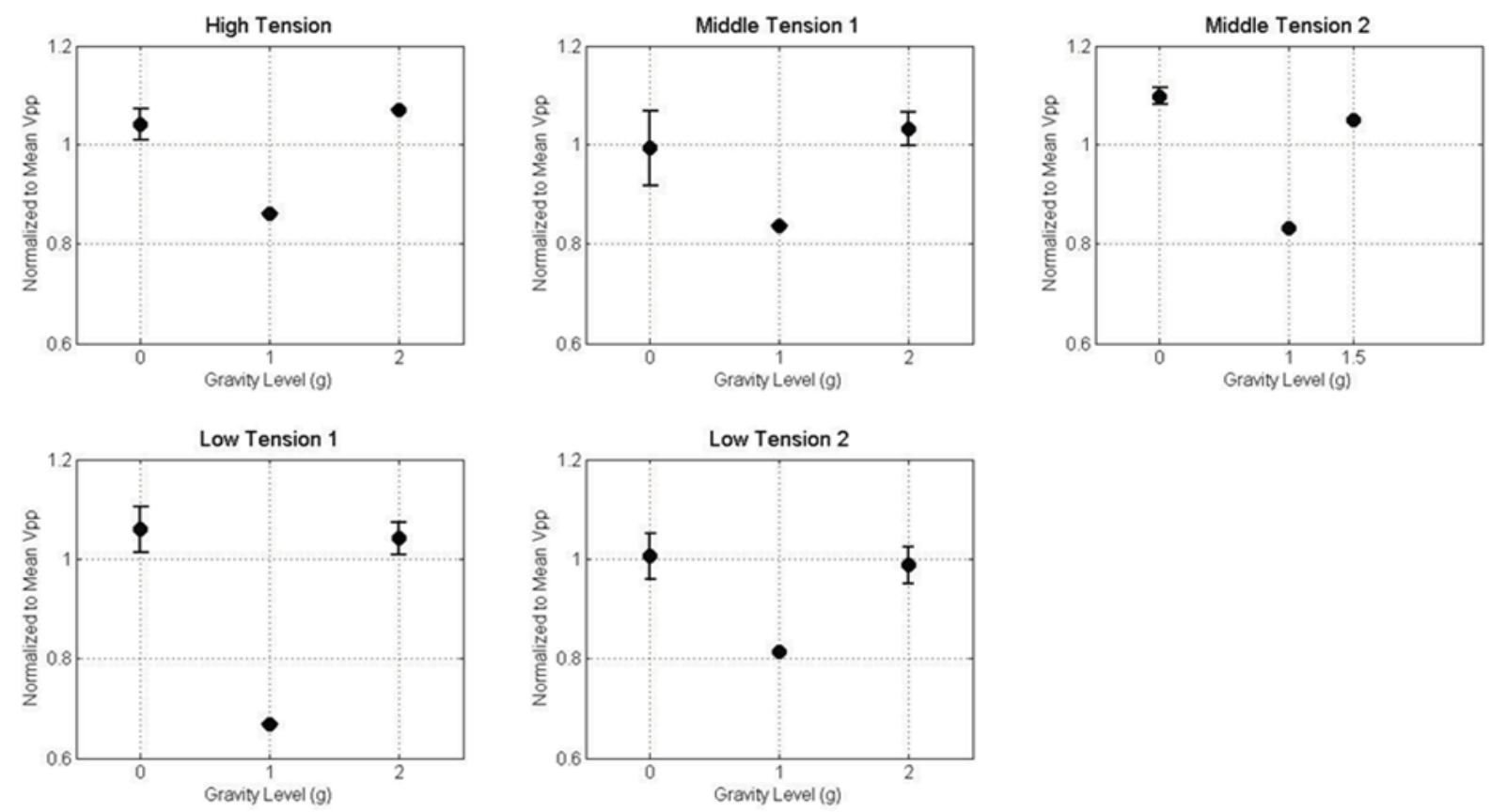

Figure 10. Parietal rubber holder and elastic strap headgear traces (flight day 28 February 2013): Normalized AC signal intensity measured over multiple parabolas plotted vs. gravity level for each tension level. 

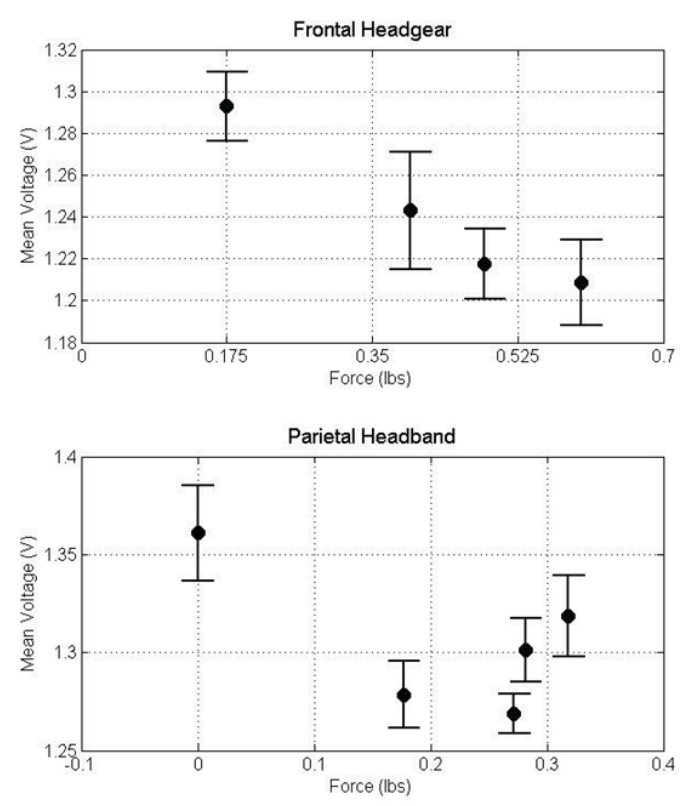

Figure 11. AC intensity values averaged across gravity levels, plotted as a function of force against the head phantom material.

Top: Frontal semi-rigid band (flight day 1 ). Bottom: Parietal rubber holder (flight day 3 )
AC signal intensity variability analysis results:

Better headgear attachment mechanisms will show less variability of signal intensity across $\mathrm{g}$ levels. The variability in the Frontal semi-rigid band (Fig 9.) is less than that seen with the Parietal rubber holder with elastic straps (Fig.10). Therefore, this portion of the analysis implies the frontal rigid headband may be superior in maintaining comparable intensity levels across different values of acceleration (although not across transitions in g-level).

Overall changes in intensity depending on tension and independent of gravity level were observed (Fig. 11). At low tensions, higher signal intensity may be due to greater exposure to ambient light. This result occurs because at high tensions, the mechanical coupling at the surface is tighter, allowing less ambient light into the signal. Such mechanical coupling methods allow the dark baffling material around the optical components to reject undesirable light that is not going into or coming out of the gray matter phantom (the tissue). This baffling effect may account for the decreasing intensity with increasing tension for the frontal headgear. However, this effect must be investigated further as the $a c$ signal analysis was limited to short time, high data rate collection windows.

\section{Conclusions}

The variable gravity flight objectives were built around our goal to improve headgear and instrumentation for performing Functional Near Infrared Spectroscopy (fNIRS) in operational aviation and space environments. The performance of the elastic and spring-mounted attachment mechanisms comprising the coupling force of our custom headgear designs was assessed and confirmed well for reasonable strap tensions. Some unexpected lessons were learned as well during this flight opportunity. Our overall observations, conclusions and recommendations include the following points.

fNIRS is a non-invasive optical neuroimaging technique used to quantify functional activation of the brain regions underlying the optical probes. Time traces of optical intensity changes utilized to calculate hemoglobin concentration can easily be confounded by systemic physiology arising from blood pressure oscillations, respiration and the cardiac cycle. When performing fNIRS measurements, probe coupling force and motion on the head also interferes with the proper measurement of hemodynamics in the brain ${ }^{11}$. It is therefore imperative to design and build headgear and instrumentation to directly address this interference, such that functional hemodynamic measurements of brain activation can be used to identify and maintain attentive cognitive state during safety-critical operational tasks ${ }^{12}$. To determine which aspects of our custom fNIRS headgear designs perform best under operational flight-like conditions, two headgear prototypes were assembled and tested with an fNIRS flight rig. Motion artifact in the absence of functional activation and physiological confounds were observed by using a phantom head. We presented motion-artifact-based performance metrics for each design, and demonstrate one method for the removal of such artifacts via signal processing.

Our design employing rubber holders with elastic straps performed best, despite relatively lower force between headgear and the phantom head. We conclude that our novel spring-loaded and elastic attachment mechanisms for the entire head will perform adequately under varying loads, leaving the intensity measurements less susceptible than current technology to mechanical changes or gravitational changes in the environment. The drawback is that elastic attachment may contribute to increased AC-signal variability. 
Oscillating artifacts and steep, large changes in detected intensity magnitude were observed which were not coincident with changes in force, acceleration or g levels. We believe these are the result of optical instrumentation connections and not headgear attachment mechanisms.

We noted large changes in detected intensity specifically at times of steep change in g level. We recommend that researchers assessing functional activation across transitions in $\mathrm{g}$ interpret their results and consider their hardware with care to avoid attributing changes actually due to the mechanical environment to changes due to neurophysiology.

In operational use, monitoring the acceleration of optical headgear may become a recommendation for best practices in the implementation of fNIRS. In dynamic operational environments in which headgear motion and thus signal contamination cannot be avoided, we recommend implementing the best-achievable headgear attachment mechanism to ensure good coupling to the skin and to reduce signal contributions due to motion, always in combination with post-processing artifact removal or correction.

Future work should include further ground-based clinical testing, amelioration of probe and headgear designs for comfort and reliability, the completion of an IRB for human testing and the performance of human tests in an appropriate varying gravity environment. The next logical step would be to conduct similar tests with human subjects performing a task in relevant aviation and microgravity environments. To achieve this goal, we plan to iterate and expand hardware, headgear prototypes and data processing using the lessons learned herein and results presented in this paper. We may also advance our electronics design to provide frequency-domain fNIRS capability as resources permit, or use and compare commercially-available systems. Upgrades necessarily will include laser control for safe use with human test subjects. We shall continue to work closely with all radiation safety officers and the committee for the protection of human subjects to ensure all human test subject safety requirements are met.

\section{Acknowledgments}

This research was sponsored by the NASA Glenn Research Center Aeronautics Directorate Vehicle Systems Safety Technology (VSST) program. The variable gravity flights were sponsored the NASA Office of the Chief Technologist Flight Opportunities Program. The authors also wish to thank the Johnson Space Center Reduced Gravity Office for their flight support.

\section{References}

${ }^{1}$ B. Khan, C. Wildey, R. Francis, F. Tian, M. R. Delgado, H. Liu,1 D. MacFarlane, and G. Alexandrakis, Improving optical contact for functional near-infrared brain spectroscopy and imaging with brush optodes, BIOMEDICAL OPTICS EXPRESS, Vol. 3, No. 5 (2012) 878-898

${ }^{2}$ Ferrari, M., Quaresima, V., A brief review on the history of human functional near-infrared spectroscopy (fNIRS) development and fields of application, NeuroImage (2012), doi:10.1016/j.neuroimage.2012.03.049

${ }^{3}$ S. Lloyd-Fox , A. Blasi, C.E. Elwell, Illuminating the developing brain: The past, present and future of functional near infrared spectroscopy, Neuroscience and Biobehavioral Reviews 34 (2010) 269-284

${ }^{4} \mathrm{~J}$. Gervain et al., Near-infrared spectroscopy: A report from the McDonnell infant methodology consortium, Developmental Cognitive Neuroscience 1 (2011) 22-46

${ }^{5}$ P. Cowings, Psychophysiology of Spaceflight., in 14th IAA Humans In Space Symposium, Living in Space: Scientific, Medical and Cultural Implications. 2003: Banff, Alberta, Canada

${ }^{6}$ Q. Zhang, X. Yan, G. E. Strangman, Development of motion resistant instrumentation for ambulatory nearinfrared spectroscopy, Journal of Biomedical Optics (2011) 16(8), 087008

${ }^{7}$ S. Schneider, V. Abeln, C. Askew, T. Vogt, U. Hoffmann, P. Denise, H. Strüder, Changes in cerebral oxygenation during parabolic flight, European Journal of Applied Physiology 113 (2013) 1617-1623

${ }^{8}$ R. M. P. Doornbos, R. Lang, M. C. Aalders, F. W. Cross and H. J. C. M. Sterenborg The determination of in vivo human tissue optical properties and absolute chromophore concentrations using spatially resolved steady-state diffuse reflectance spectroscopy, Phys. Med. Biol. 44 (1999) 967-981

${ }^{9}$ M. A. Kramer, R. W. Boyd, L. W. Hillman, and C. R. Stroud, Jr., Propagation of modulated optical fields through saturable-absorbing media: a general theory of modulation spectroscopy, J. Opt. Soc. Am. B/Vol. 2, No. 9 (1985), 1444-1455

${ }^{10}$ J. R. Mackey, G. Adamovsky, B. M. Floyd, P. Tin, A. R. Harrivel, Improved light injection and detection methods for fNIRS headgear for use in avionics and astronautics, 2nd Biennial fNIRS Meeting, October 26-28, 2012, London, UK, NASA New Technology Report \# 1351026054

${ }^{11}$ G. Gratton, M. R. Goodman-Wood, and M. Fabiani, Comparison of Neuronal and Hemodynamic 
Measures of the Brain Response to Visual Stimulation: An Optical Imaging Study, Human Brain Mapping, WileyLiss, Inc., (2001) 13:13-25

${ }^{12}$ T. Shimizu, S. Hirose, H. Obara, K. Yanagisawa, H. Tsunashima, Y. Marumo, T. Haji and M. Taira, Measurement of Frontal Cortex Brain Activity Attribute to the Driving Workload and Increased Attention, SAE (2009) paper NO. 2009-01-054 